\title{
The Development of Primary School Students' 3D Geometrical Thinking within a Dynamic Transformation Context
}

\author{
Christos Markopoulos' ${ }^{1}$ Despina Potari², William Boyd ${ }^{3}$, Koralia Petta', Marilyn Chaseling1 \\ ${ }^{1}$ School of Education, Southern Cross University, Lismore, Australia \\ ${ }^{2}$ Faculty of Mathematics, National and Kapodistrian University of Athens, Athens, Greece \\ ${ }^{3}$ School of Environment, Science \& Engineering, Southern Cross University, Lismore, Australia \\ Email: christos.markopoulos@scu.edu.au, dpotari@math.uoa.gr,william.boyd@scu.edu.au, \\ koralia.petta@scu.edu.au, marilyn.chaseling@scu.edu.au
}

Received 26 June 2015; accepted 7 August 2015; published 10 August 2015

Copyright @ 2015 by authors and Scientific Research Publishing Inc.

This work is licensed under the Creative Commons Attribution International License (CC BY).

http://creativecommons.org/licenses/by/4.0/

(c) (i) Open Access

\section{Abstract}

This study examines children's thinking about geometrical solids through an investigation of dynamic transformations employed by young children making mental transformations of an orthogonal parallelepiped. The focus of the study is on the investigation of the role that a dynamic environment could play in the development of children's geometrical thinking concerning geometrical solids and their properties. Twenty 6th grade children, who had previously worked with dynamic transformations of physical models of geometrical solids in their classroom, were interviewed. Analysis of the data resulted in a categorization of children's thinking, and indicated a development from a perceptual to a geometrical consideration of the solid. Although not all the children reached an advanced level of thinking, the context of dynamic transformations promoted the development of most children's geometrical thinking. There is also an indication that children's experience with dynamic transformations of physical models in a mathematics classroom environment can act to allow children to transfer experience to the context of mental transformations.

\section{Keywords}

3D Geometry, Visualization, Mental Imagery, Geometrical Thinking, Dynamic Transformations

\section{Introduction}

A number of research studies have focused on children's thinking on three-dimensional solids. Most appear to

How to cite this paper: Markopoulos, C., Potari, D., Boyd, W., Petta, K., \& Chaseling, M. (2015). The Development of Primary School Students' 3D Geometrical Thinking within a Dynamic Transformation Context. Creative Education, 6, 15081522. http://dx.doi.org/10.4236/ce.2015.614151 
be on the nets of solids (Mariotti, 1989; Potari \& Spiliotopoulou, 1992; Stylianou et al., 1999; Cohen, 2003), on their plane representations (Cooper \& Sweller, 1989; Mitchelmore, 1980; Ma et al., 2009) and on constructions of the solids by unit cubes (Bishop, 1985; Battista \& Clements, 1996; Sack, 2013). In most studies, children's thinking about geometrical solids was studied in the frame of van Hiele's theory (1986) and the focus was to see the suitability of the levels in this area. Pegg (1997), for example, extended van Hiele's work by highlighting the differentiation between Levels 2A and 2B in the case of three-dimensional solids. The research on this domain exploits this theory and uses it as a way to interpret and classify children's thinking using methods such as questionnaires, test or interviews (Lawrie et al., 2002; Pegg \& Baker, 1999).

The above studies are mainly exploratory and do not indicate the conditions under which children's thinking on three dimensional solids develops. In this paper, we investigate the development of children's thinking concerning geometrical solids while they transform them mentally. The children had experiences in transforming geometrical solids using physical materials and computer representations in a classroom teaching experiment. These contexts are characterized by the dynamic manipulation of geometrical solids, a process where the solid changes its form through the variation of some of its components and the conservation of others. The analysis of the 3D geometrical solids into component parts follows a similar approach with the research study by Ambrose and Kenahan (2009). In this study, we investigate 6th grade children's geometrical thinking about geometrical solids and their properties in a context of the mental transformations. This context is related to the children's visualization process that involves the recall or the construction of the solid's mental image, its representation and its appropriate transformation if necessary (Brown \& Wheatley, 1997).

\section{Theoretical Framework}

\subsection{The Concept of Geometrical Solid and the Process of Transformation}

The geometrical solid is an abstract entity governed by mathematical relations and theories. A controversial epistemological issue is the relationship between a real object and a mathematical one. Waldegg (2004) indicates that, by using an example from the history of mathematics, the absolute differentiation of these two worlds - the mathematical and the real—acted as an obstacle for the development of geometry. Fischbein (1993) also states that, "the complete independence of mathematics, as a closed world of formally postulated entities, has proved itself to be an impossibility” (p. 16). Mathematics education research aims to find ways that support children to construct these abstract relations and theories by reflecting on their experiences with real objects. These ways either emphasize the use of physical materials (Sdrolias \& Triandafillidis, 2008), real world metaphors (Lakoff \& Nunez, 2000) or computer representations of geometrical objects (Mariotti, 2000; Jones, 2000; Arnold \& Lawson, 2003).

Battista (2007), in reviewing several theories relevant to geometric learning, associates the theory of abstraction to the van Hieles theory of geometrical thinking. He suggests a transformational perspective on building interrelationships between components of the geometrical shapes. In particular, the first van Hiele level is associated to the internalized level of recognizing and visualizing the shapes as whole entities. In the second van Hiele level, shape recognition is based on the interrelationships between the shape and its components. It prerequisites the interiorized level of abstraction and, therefore, the decomposition of the shape to its components and the establishment of interrelationships between the components of the shape. The role of the dynamic transformation is crucial, as it supports these processes of moving from the recognition of the whole shape to its decomposition, the recognition of its components and finally the construction of interrelationships between the components by operating on them.

This perspective is similar to Duval's (2006) analysis of the role and the cognitive functioning of visualization. Duval associates the visual recognition of any figure with its shape and its topological configurations, according to the principles stated by the Gestalt theory. However, the proposed "operative apprehension" of a geometrical object is associated with its decomposition in all the figural units according to their dimension: $1 \mathrm{D} / 2 \mathrm{D}, 2 \mathrm{D} / 2 \mathrm{D}$, $3 \mathrm{D} / 2 \mathrm{D}$, and the $0 \mathrm{D} / 2 \mathrm{D}$ that cannot be perceptively discriminated.

\subsection{The Analysis of the Concept of Geometrical Solid}

The epistemological analysis of the concept of geometric solid we present is based on "logic of significance", which introduces the Piaget \& Garcia (1989), rather than a traditional, logical analysis. According to Piaget's 
theory, the action and the motion are considered primary evidence for establishment of mathematical concepts by the student. Thus, the analysis of a geometric solid is based on possible actions and cognitive functions of pupils about the particular mathematical meaning. The cognitive functions are mental actions necessary to construct mental schemes, which are possibly, but not necessarily, associated with a particular object.

Piaget's model is extended and enriched by the theory of Freudenthal (1991) about the mental structures. In particular, mathematical concepts are described as cognitive structures, which can be classified as "poor" and "rich". A mathematical concept is treated as a cognitive structure in various forms. More specifically, the combinatorial structure of the polyhedron includes polyhedron as a structure consisting of the components such as the edges, the faces, and the vertices. On the other hand, the metric structure of a polyhedron is richer than the combinatorial one, and includes the metric properties of the solid such as the measure of lengths, angles or other components, and the parallelism of edges or faces. Even a richer structure of polyhedron could go beyond the measurement and comparison of sizes, and include parallelism and collinearity. The latter is the homoparallel structure of the polyhedron. Without the parallelism, the collinearity refers to a richer structure of polyhedron, the projective one. In a similar way, Duval (2011) discusses a mathematical way of perceiving a figure, distinguishing the global figure from its figural units. The figural units can be discriminated according to their dimension-0D, 1D, 2D and 3D-and their discrimination is a prerequisite for conceptualizing geometrical relationships and developing geometrical reasoning.

The analysis of the concept of geometrical solid is based on three dimensions:

- its components that contribute to the formation of the solid;

- the properties of the solid; and

- possible transformations of the solid through the variation of its properties.

Such an analysis is considered appropriate, as it seems compatible with the way students build their understanding of the concept of the geometrical solid. The concept of the solid is considered as a network of relationships between its components and their properties, and therefore a systemic network methodology was used (Bliss et al., 1983).

Figure 1 illustrates the systemic network of the analysis of the concept of the geometrical solid. In the systemic network two types of brackets have been used. The curly brackets ( $\{$ ) and the square brackets ([) define the association and the independence of the subsequent categories respectively. Figure 1 demonstrates that the concept of the geometrical solid is analyzed in terms of its form, the properties of its components, and its potential transformations. These three categories are not independent, but are associated (hence enclosed in curly brackets), while the square brackets distinguish the form of the solid into the two exclusive categories, "solid as a whole" and "solid according to its components".

\subsubsection{The Components of the Geometrical Solid}

Students could experience the solid merely as a whole by focusing only on its form. In addition, the components of a solid, such as vertices, edges, faces, 2D and 3D angles, heights, area and volume, may be the subject of study. These components constitute, by themselves, geometrical concepts, each of which could be studied and analyzed separately. These concepts can be characterized as zero-dimensional (vertices), one-dimensional (edges, height), two-dimensional (faces, 2D angles and area) and three-dimensional (volume and 3D angles). The classifications of these geometric concepts according to the dimensions to which they relate, in combination with their further mathematical analysis, make evident the complexity of the mathematical concept of the geometric solid. Thus, for example, the edges are both the "building blocks" of the solid and components of the geometric concept of the face of the solid.

\subsubsection{Geometrical Solid's Properties}

The properties of a solid describe the way that its components correlate to each other. These properties could be in reference to the solid itself or to other solids. In addition, the properties of the components (e.g. the number of faces), the relations among the same type of components (e.g. the equality of edges) and the relations among different types of components (e.g. angles and edges) constitute the structure of the geometric solid. Although the components of any polyhedron are the same, variations of their properties differentiate the specific categories of polyhedron. The edges are components of both cubes and rectangular boxes, for example, although while the number of edges is the same in both, their sizes differentiate the two.

The three kinds of properties, size, number and form, however, do not relate to all types of components. Size 


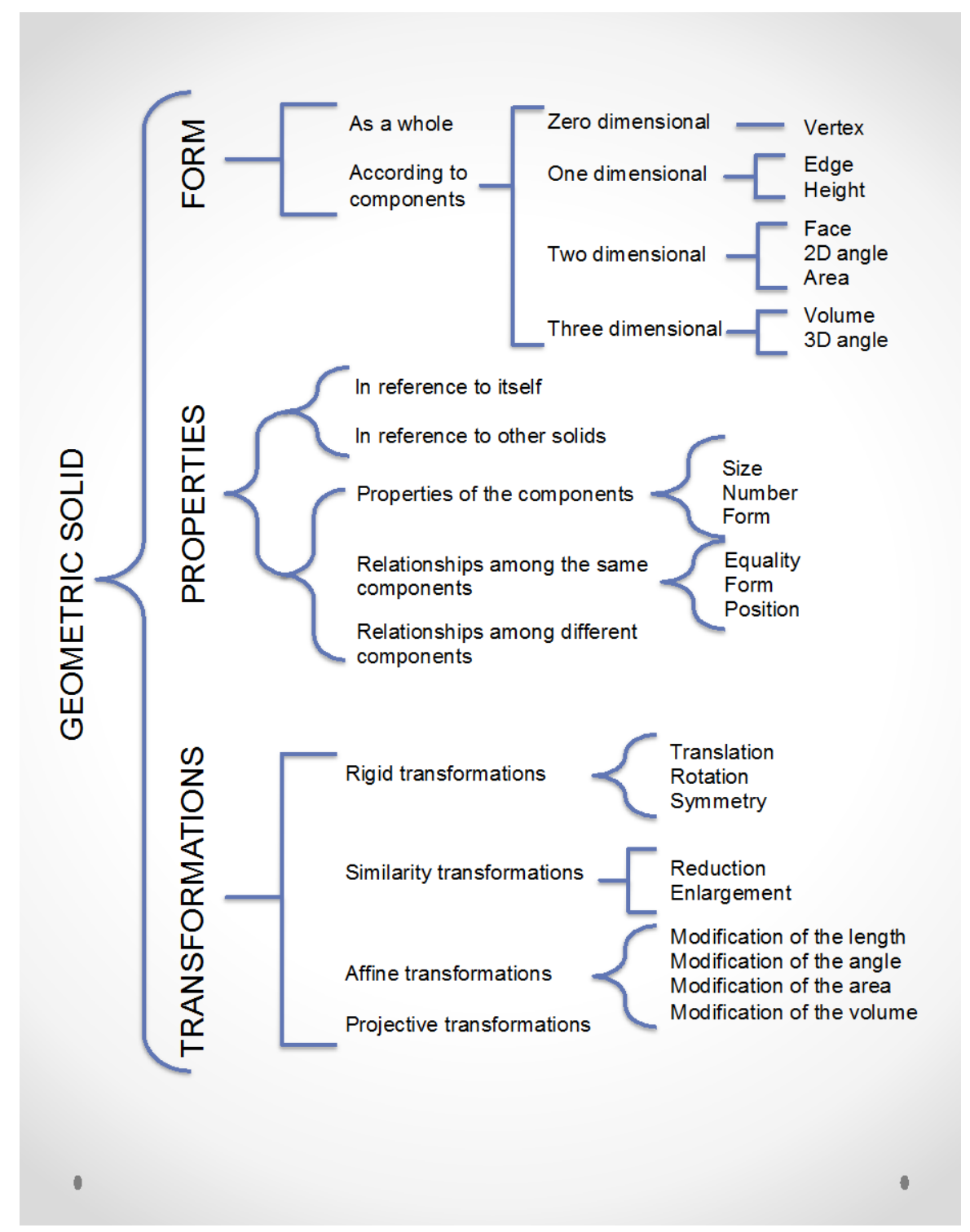

Figure 1. Analysis of the concept of geometrical solid.

refers to the edges, the height, the angles (2D and 3D), the area and the volume, number relates to the vertices, the edges, the faces, the heights and the angles, while form relates only to faces and especially to the shape of each face. These properties do not include how these are actually related to each other. The number and the size of the edges, for example, do not imply a possible relationship of equality or parallelism between them. Thus, the comparative relationships between components of the same kind are necessary to show how the components with their special characteristics contribute to the formation of each polyhedron. These relationships include comparing sizes, comparing forms and comparing positions. In particular, the correlation of the same components based on their size results in the discovery of equality or inequality between all or certain of them. Moreover, the size comparisons are applicable to components such as edges, faces but not to vertices. The comparison of the form refers to the association between the faces of solid based on the shape of each face and it is complementary to the size comparisons. Finally, comparative relationships between the same components may be based on the relative position of each element; two edges of a polyhedron can be, for example, parallel, intersected, or asymptotic. The components that can be studied in relation to their positions are the edges, heights, angles and faces of a solid.

The third category of the solid's properties refers to associations between properties of different components, 
highlighting their interdependence in the creation of the polyhedron. Thus, we can discern relationships among properties of two different components, but also relationships among more: what way, for example, does the equality of a cube's edges affect the equality of the faces, and vice versa?

\subsubsection{The Transformations of the Geometrical Solid}

The analysis of the types of transformation is based on the concept of invariance (Otte, 1997). The transformation of a solid is possible through the variation of some of its properties and the conservation of others. Thus, the geometrical solid is associated with a group of transformations that leave in each case a number of its properties invariant (Modenov \& Parkhomenko, 1965). Any transformation of the solid could be considered as a process including the mapping of the initial solid into the last one.

Rigid transformations leave invariant the properties of the solid's components while changing its location in space and possibly its orientation. Thus, this type of transformation could be considered as the mapping of the solid from its initial position to its final one. The fundamental types of rigid transformation of a solid on space are translation, rotation and reflection.

Translation transformation includes the mapping of each point of a solid to another point within a fixed vector. The rotation of a solid presupposes the line of rotation, which can be any straight line in space, either through the solid or not, and the direction angle in which each point of solid rotates around the line. Finally, reflection is the rigid transformation where each point of solid reflects to its symmetrical point through either a plane or an axis or a point.

While the solid that results from a rigid transformation maintains both the shape and the dimensions in relation to the initial solid, similarity transformations preserve that form of the solid. Similarity transformations result from the reduction or the enlargement of the solid by the variation of the size of the edges and the heights in the same ratio.

Affine transformations preserve the parallelism and the collinearity of the components of the solid, and change the size of the edges, the heights, the angles (2D and 3D), the area and the volume of the solid. In this type of transformation, the initial solid differs with regard to the form from the final solid, although some of its properties could remain invariant. The transformation of cube into a rectangular parallelepiped, by increasing the size of the edges of the cube, is an example of affine transformation.

Projective transformation results in the alteration of a solid's parallelism in addition to the size of its edges, angles, area and volume. Thus, in the affine transformation the parallelism as a property of the components does not exist, and consequently a pair of parallel edges may possibly be converted to two edges that are not parallel.

\subsection{Visualization and the Dynamic Transformation of the Geometrical Solid}

The development of visualization and the reasoning based on mental images are associated with the improvement of geometrical thinking (Lean \& Clements, 1981; Wheatley, 1990; Hershkowitz, 1989). Reasoning based on visualization is founded on the use of visual space elements that can be either mental or physical (Gutierrez, 1996; Presmeg, 2006). Wheatley (1990) considers that this process includes the construction of a mental image, its representation when it is necessary, and its appropriate transformation. Particularly, it is considered that when the recall of an already constructed mental image is necessary, this can be through its representation. The representation of the mental image may not take the exact form of that originally constructed and probably a transformation is needed. For example, an initial mental image of the rectangular parallelepiped probably shows a box with two square faces. In the case, however, of recalling the mental image of this particular solid in a problem solving situation whereas where the rectangular parallelepiped does not have square faces, a dynamic transformation of the image is necessary. The process of visualization, therefore, refers to the creation, the representation and the dynamic manipulation of mental images, although their simultaneous occurrence is not necessary (Wheatley, 1990). The extent to which this process is considered to contribute to the development of mathematical thinking depends on whether it leads to abstraction and generalization. Considering these assumptions, we suggest that a dynamic transformation of a solid includes the initial solid, the process of transformation, and the final solid.

\subsection{The Development of Geometrical Thinking about Geometrical Solids}

The development of students' conceptions about the concept of geometric solid could be associated with the 
stages of development of geometric thinking, as proposed in van Hieles' theory. The first four stages of concerning students' geometric ideas in primary and secondary education (Lawrie et al., 2000), expanded (stages 2A and 2B) following Pegg (1997) are:

Stage 1: Students perceive the solid holistically, and fail to focus on its components. The identification of the solid, and the distinction between different solids are possible, but they are not based on the properties of each solid, even if specific components of the solid, such as edges, vertices and faces are mentioned.

Stage 2A: Students recognize the solid on the basis of a specific property and not overall form. The properties of the solid on which the recognition is based include correlation between a particular type of components (e.g. the orthogonal parallelepiped is associated with equality of opposite faces). Students do not necessarily ignore the distinction between other components, and may refer to them, but do relate properties between components.

Stage 2B: Components and their properties are treated as independent characteristics of the solid. Students refer to properties such as angle size, angles and edge equality, but do not relate these to each other.

Stage 3: Students recognize the potential of the solid's properties to develop its form. Students develop reasoning that includes the relations between the properties of the solid and itself, and, based on these relations, between different solids.

Stage 4: Students develop their ability for analytic reasoning. Their reasoning is based on a geometric proof, by determining essential and necessary conditions. Correlations between the solid and its properties, and between different solids, are structured as definitions for the meaning of geometric solids.

\section{Methodology}

The research method adopted in this study was the clinical interview. The clinical research methods are based on the principles of constructivism and aim to investigate children's conceptions. The researcher acts as a teacher interacting with the children while aiming to investigate their thinking. By reflecting on these interactions, the researcher tries to interpret the children's actions, and forms hypotheses concerning these conceptions. These hypotheses are evaluated and consequently verified or revised (Bell, 1993; Hunting, 1997).

Participants: Twenty 6th grade (year 6) school children (11 years old) from three different non-streamed classes, in three different public primary schools, volunteered to participate in the present study. These students were predominately from medium and lower social economic backgrounds and resided in a medium-sized city in Greece. Before this study, these children had participated in a classroom teaching experiment, along the same lines described by Cobb, Yackel and Wood (1992). The classroom teaching experiment provided an alternative approach to the official mathematics curriculum integrating dynamic representations of the geometrical solids (Markopoulos \& Potari, 2000; Markopoulos, 2003).

The process: The children were interviewed individually for about an hour on a number of tasks similar to those they had faced in the classroom, but without visual reference. The context of the activity differed, as neither dynamic models nor dynamic computer representations were used. The tasks were not predefined, but developed according to children's responses, although each interview followed a common structure. Children were asked to:

- imagine and describe an orthogonal parallelepiped;

- suggest a mental dynamic transformation of the solid; and

- focus on the mental dynamic transformation.

Each student was asked to imagine an orthogonal parallelepiped and describe it. Then students were asked to suggest and perform a mental transformation of the solid through the dynamic change of its properties. Finally they were asked to study the varied properties of the initial solid. A dynamic transformation of a geometrical solid involves three elements: the initial solid, the process of the transformation, and the resulted solid (Figure 2). The study of the dynamic transformations depends on which of these three elements is the focus of attention. The dynamic transformations that took place during the interviews took an orthogonal parallelepiped as the initial solid. The children were asked to transform this solid, mentally defining either the process of the transformation or the resulting solid. Students attempting to describe their mental actions sought visual referents, and they were allowed to refer to real objects to facilitate their descriptions.

Data analysis: The data comprised twenty transcribed video recordings. The transcriptions were coded to yield categories representing stated aspects of the children's geometrical thinking. The data for each child was also examined to yield evidence for possible development of the child's thinking. 

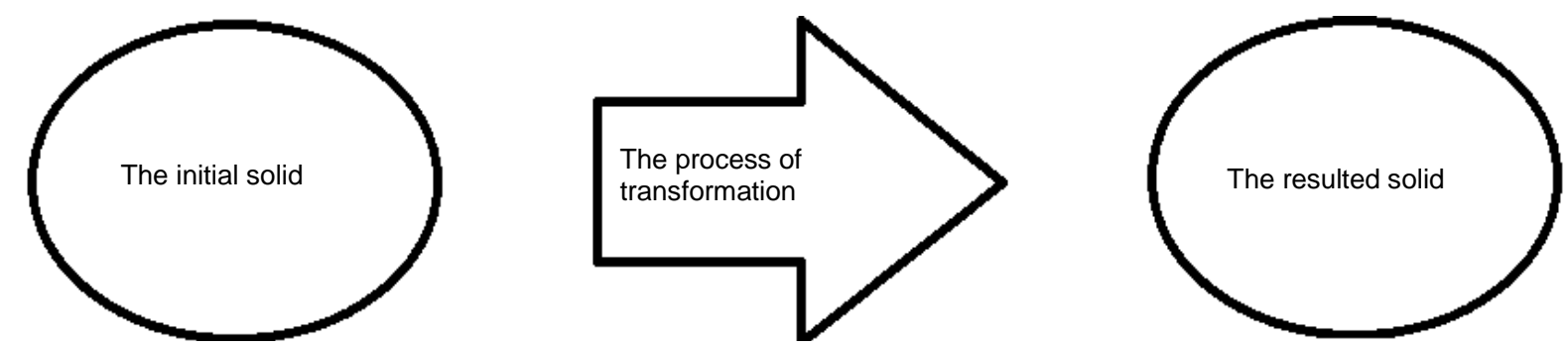

Figure 2. The three elements of the transformation.

More specifically, the analysis of the interviews commenced with a classification of students' understandings of the concept of geometric solid as elaborated in the process of mental dynamic transformations of the solid. Records of the mental dynamic transformations experienced by each student were based on a transformation of a solid orthogonal parallelepiped; students are asked to mentally transform the orthogonal parallelepiped by describing either the transformation process itself or the resulting solid.

\section{Results}

\subsection{The Type of Transformations}

From the initial analysis of the transcribed interviews, two main types of transformation were identified (Table 1).

a) Dynamic transformation of the solid through the modification of its properties to create a different solid. Children who attempted this kind of transformation defined the process of transformation by specifying the properties and size of the components - edges, faces and angles - they wanted to modify. A child might, for example, suggest the modification of one dimension, the height, and modify it by increasing its length.

b) Specification of the resulting solid. The target solid was either an orthogonal parallelepiped with two square faces or a cube. Although this type of transformation required the child to anticipate the specific modified properties, a rather conceptually complex process (Markopoulos \& Potari, 1999, 2000), most children carried out this type of transformation (eighteen of the twenty pupils). This tendency probably reflects the children's classroom experiences in manipulation of dynamic models. However, as it will be illustrated below, the degree of awareness of the modification varied amongst them.

The interviews reveal that students' conceptions of the process of a mental transformation include, initially, the definition of those properties of the solid that are directly or mentally changed. In both dynamic physical and computer models, the directly-changed properties were the properties that students themselves had modified. For mental transformations, the directly-modified properties are not the result of students' physical actions, but of their mental actions. The direct mental changes indicate that the directly-changed properties vary in relation to the type of proposed transformation. In particular, type A directly-changed properties refer to the specific properties that students modified to transform the orthogonal parallelepiped into a different solid. For type B transformations, the directly mental changes transform the initial solid into an orthogonal parallelepiped in a prototypical form or into a cube.

Apart from investigating and determining the directly modified properties, the study of dynamic transformation includes exploration of the properties that changed indirectly. The directly changed properties affect other properties of solid and, consequently, modify them. The distinction of modifications into direct and indirect are associated with the correlation between the properties of the components of the geometrical solid. The dynamic transformation of an orthogonal parallelepiped may be made, for example, through the modification of the solid's height while maintaining the edge lengths. A direct modification of height results to several indirect changes in other properties of different components, such as, the change of the two faces, angle sizes, surface area and volume. The students' conceptions of the interrelations of component properties include, therefore, the determination of the impact of the direct modification of the height to the remaining properties.

\subsection{Emerging Categories of Children's Geometrical Thinking}

In relation to the children's thinking about geometrical solids, four categories emerged. 
Table 1. The types of transformation identified in interviews with children asked to transform an orthogonal parallelepiped.

\begin{tabular}{|c|c|c|c|c|c|c|c|}
\hline \multirow[b]{2}{*}{ Child } & \multirow[b]{2}{*}{ No. of transformations } & \multicolumn{4}{|c|}{ A. Modification of properties } & \multicolumn{2}{|c|}{ B. Description of resulting solid } \\
\hline & & A1 Edges & A2 Faces & A3 Dimensions & A4 Angles & $\begin{array}{l}\text { B1 Orthogonal parallelepiped } \\
\text { with two square faces }\end{array}$ & B2 Cube \\
\hline St1 & 2 & & & & $\sqrt{ }$ & $\sqrt{ }$ & \\
\hline St2 & 2 & & & & $\sqrt{ }$ & & $\sqrt{ }$ \\
\hline St3 & 2 & & & & $\sqrt{ }$ & & $\sqrt{ }$ \\
\hline St4 & 2 & $\sqrt{ }$ & & & & & $\sqrt{ }$ \\
\hline St5 & 2 & $\sqrt{ }$ & & & & & $\sqrt{ }$ \\
\hline St6 & 1 & & & & & & $\sqrt{ }$ \\
\hline St7 & 2 & & & & & $\sqrt{ }$ & $\sqrt{ }$ \\
\hline St8 & 2 & & $\sqrt{ }$ & & & & $\sqrt{ }$ \\
\hline St9 & 1 & & & & & $\sqrt{ }$ & \\
\hline St10 & 2 & & & & & $\sqrt{ }$ & $\sqrt{ }$ \\
\hline St11 & 1 & & & $\sqrt{ }$ & & & \\
\hline St12 & 2 & & & & & & $\sqrt{ }$ \\
\hline St13 & 2 & & & & & $\sqrt{ }$ & $\sqrt{ }$ \\
\hline St14 & 2 & & $\sqrt{ }$ & & & & $\sqrt{ }$ \\
\hline St15 & 1 & & & & & $\sqrt{ }$ & \\
\hline St16 & 2 & & $\sqrt{ }$ & & & & \\
\hline St17 & 1 & & & & & & $\sqrt{ }$ \\
\hline St18 & 1 & & & & & & $\sqrt{ }$ \\
\hline St19 & 1 & & & & & & $\sqrt{ }$ \\
\hline St20 & 1 & & & & & & $\sqrt{ }$ \\
\hline
\end{tabular}

C1. The solid conceived as a total entity, with properties not identified. Although the dynamic transformation of a solid involved the modification of its properties, the children studying the transformation identified the two solids holistically, and limited their description to the name of each solid. The description of an orthogonal parallelepiped included, for example, metaphors based on the solid's visual appearance and not its properties: "it looks like a box".

C2. Geometrical thinking based on comparison between modified properties. The solid is not considered as a total entity but is related to the properties modified directly by the children during transformation. When modifying the edges of an orthogonal parallelepiped to get a cube, for example, children compared the two solids in reference to this particular property.

C3. Correlating properties of the solids. The children related the properties they had modified to the solid, but without realizing the role of the subsequent property modification in the formation of the new solid. While the children modified edge lengths of an orthogonal parallelepiped, for example, they did relate this change to subsequent changes to face area. They could not realize the role, also, of subsequent modification to in the process of transforming the initial solid into a different one.

C4. Building relationships between direct and modified properties and the geometrical solids, and between the solids themselves. When transforming an orthogonal parallelepiped into a cube, for example, the children related the direct modified changes in edge length to shape and face area, and also to the resulting solid.

These four categories of students' geometrical thinking around the concept of geometrical solid through its dynamic transformation can be related to the Van Hieles level of geometrical thinking with regard to the dis- 
crimination between level 2A and 2B (Pegg, 1997; Lawrie et al., 2000).

\subsection{The Development of Geometrical Thinking}

The children's thinking shifted during the interview with reference to the type or types of transformation that each child performed (Table 2).

Students represented a range of developmental stages and evolution. To close the paper, we discuss four representative examples, drawing attention to matters that emerged concerning the development of children's geometrical thinking and the role of the transformations. In particular, we analyse four examples of students who reached different levels of development by the end of the experiment.

- Remaining at a holistic level. Student St8 did not evolve reasoning beyond the first category (C1).

- Starting to recognise the modified properties. Student St12 moved towards recognition of the specific modified properties (C2).

- Realising the changes in the properties. Student St13 reached the third category (C3).

- Building relations among the solids based on the modified properties. Student St3's thinking developed to the final category (C4).

\subsubsection{Remaining at a Holistic Level}

St8 described initially the rectangular parallelepiped by pointing to a physical referent, the chalk box: "It looks like the chalk box". Later in the interview, he referred to the dimensions, angles, number and shape of faces. However, his descriptions were rather general—_It has length, width, height”; "They are wide”; "[the faces] are

Table 2. The children's development of geometrical thinking, as identified during the interviews, detailing the types of transformation that each child experimented with, and categories of thinking for each transformation type.

\begin{tabular}{|c|c|c|}
\hline Child & Types of transformation & Category of thinking \\
\hline St1 & A, B & C1-C2, C2-C3 \\
\hline St2 & A, B & C2-C3, C3-C4 \\
\hline St3 & A, B & C2-C4, C3-C4 \\
\hline St4 & A, B & C1-C2, C2-C4 \\
\hline St5 & A, B & C2-C3, C2-C4 \\
\hline St6 & $\mathrm{B}$ & C1-C3 \\
\hline St7 & $\mathrm{B}, \mathrm{B}$ & $\mathrm{C} 1, \mathrm{C} 1$ \\
\hline St8 & B & $\mathrm{C} 1$ \\
\hline St9 & B & $\mathrm{C} 2-\mathrm{C} 3$ \\
\hline St10 & $\mathrm{B}, \mathrm{B}$ & C1-C2, C1 \\
\hline St11 & A & C1-C3 \\
\hline St12 & $\mathrm{B}, \mathrm{B}$ & C1-C2, C2 \\
\hline St13 & B, B & C1-C2, C2-C3 \\
\hline St14 & A, B & C2-C4, C2-C3 \\
\hline St15 & B & $\mathrm{C} 2-\mathrm{C} 4$ \\
\hline St16 & 2 & $\mathrm{C} 1, \mathrm{C} 1$ \\
\hline St17 & B & C1-C3 \\
\hline St18 & B & $\mathrm{C} 2-\mathrm{C} 4$ \\
\hline St19 & B & C2-C3 \\
\hline St20 & B & $\mathrm{C} 2-\mathrm{C} 4$ \\
\hline
\end{tabular}


squares... they are rectangles"-or indicated difficulties in recognizing the solid's components. He talked, for example, about the angles but pointed to the vertices. It seems the student used geometrical terms but not necessarily in a meaningful way.

Consideration of the context of the task and the corresponding mental images the student constructed during the activities (Table 3) reveals two main problems that the student considered-a 2D transformation of a rectangular face to a square one, and a 3D transformation of a rectangular parallelepiped to a cube. Working in 2D and 3D seems to be independent contexts for this student. When this student was asked to mentally transform the rectangular face to a square one, he used a dynamic image based on the form of the shapes. By reflecting on the similarities and differences of the two shapes, he considered the square as the result of a transformation of a rectangle. Some of his responses were, "This is a square because it is not too long" and "It becomes square as the angles go inside". In his attempts to describe the differences between the two shapes, language was an obstacle to communicating his ideas at a mental level. He used the term "angle", for example, to indicate the side. To clarify his meaning, the researcher asked him to draw a rectangle and a square, and to compare them. However, even in this case, the comparisons remained at a holistic level. The student spontaneously used the transformation as a tool to describe the intuitive comparisons he made: "We closed the angles", meaning that the vertices moved. When the researcher asked him to place the square over the rectangle and to compare them, the student referred to their sides. His comparisons were based, however, on the perceptual differences of the two shapes, and he did not recognise the equality of their sides.

Researcher: Why do we call this a rectangle and not a square?

St8: Because we have a difference in width.

$R$ : And why don't we call it a square?

St8: Because we can make the width smaller.

In the transformation of a rectangular parallelepiped to a cube, the student constructed the image of the target solid, the cube, by focusing on the variation of the faces. Initially, he mentally transformed the front face from a rectangle to a square. Then, by relating the produced solid to the image cube, he gradually transformed the other faces of the rectangular box. The following extract indicates his approach. The student had already varied the front and top faces of the rectangular box to squares. The researcher prompted him to think about other changes.

$R$ : What else do you want to do?

St8: To make these squares [pointing to the side face of the rectangular box]

$R$ : How are you going to do this?

St8: We want to open this [pointing to the side face and "moving" it to the back].

$R$ : What is it going to change to the solid?

St8: This will become bigger and it will become square [the side face].

Later in the discussion, the student lost track of these transformations and, although he had formed the cube, he could not describe the changes that had occurred. His descriptions remained at a perceptual level, and he did not refer to any variation of the properties.

Overall, the transformation task seemed to be a supportive tool for the student to make comparisons as he used it to describe dynamically the differences between the geometrical object both in 2D and 3D. Finally, the student related the solid to its faces but only in terms of their forms, although, the student could not develop reasoning beyond a holistic consideration of the solid.

\subsubsection{Starting to Recognise the Modified Properties}

Student St12 started by considering the solids in a holistic way, but moved towards recognition of the specific

Table 3. The development of student St8's mental processes in relation to the task.

\begin{tabular}{l}
\multicolumn{1}{c}{ The context of the transformation task } \\
\hline Working in 2D \\
$\begin{array}{l}\text { - Mentally transforming and comparing a rectangular face to a square } \\
\text { - Designing and comparing a square and a rectangle }\end{array}$ \\
Working in 3D \\
\begin{tabular}{l} 
- Transforming a rectangular box to a cube \\
\hline
\end{tabular}
\end{tabular}


modified properties. He used only one type of transformation (B) and reached the second category (C2). He initially imagined a rectangular parallelepiped of a prototypical form with two square faces. He recognised the number and the shape of the faces. The context of the task changed to 2D when the researcher asked the student to compare one rectangular face to a square one. In this context, the student talked about the equality of the sides and the angles of a square and the equality of the opposite sides in the case of the rectangle. The student, studying the two geometrical shapes as independent objects, seemed to relate them to their critical properties.

Changing the context to 3D, the student chose to transform the rectangular box to a cube. To achieve this, he proposed the dissection of the solid in order to halve its rectangular faces, a static process which did not involve the expected dynamic variation of the solid's properties. In his initial attempts to justify his suggestion, he conceived the geometrical solid at a holistic level as he was only looking for a prototypical image (half of a rectangle is a square). Towards the end of the discussion, he related the modified properties, the rectangular faces, to the target solid, the cube.

$R$ : Why do you want to cut it in the middle? What would change in the rectangular faces?

St12: They would become squares.

$R:$ Why?

St12: Because we want to make a cube.

$R$ : And what would happen to the faces when it will be a cube?

St12: They will be equal.

Then, the researcher asked the student again to explain the reason why he wanted to cut the rectangular box in the middle. The student replied that, "if we take one cube and another cube we get a rectangle".

Later in the interview, the researcher tried to challenge the student's prototypical conception that half of the rectangle is a cube by suggesting another rectangular box with all its three dimensions different. Initially the student talked again about cutting the solid in the middle. Then the researcher gave him a concrete rectangular box with all its three dimensions different, and asked him to imagine how he could make it a cube. The student developed his approach of cutting the solid in the middle further. He suggested cutting it three times in order to form a cube. He also referred to the shape of the faces: "I am going to make them squares". In the end, he managed to divide mentally the solid into small unit cubes. He repeated the process continuously until he produced twenty-four unit cubes by dissecting the solid into four equal rows and six equal columns. He characteristically said: "I made four times six which equals twenty-four".

Overall, the student, in the end, related the solid to its faces through a dissecting transformation of the initial solid to a cube. In both 2D and 3D contexts, he conceived the geometrical objects as different entities. However, comparing the initial and the produced solid, he could easily move between 3D to 2D contexts. Working in 3D context, he built relations only between the spatial and the 2D elements and not with the 1D ones.

\subsubsection{Realising the Changes in the Properties}

Student St13 proposed the transformation of the orthogonal parallelepiped into an orthogonal with two square faces and a cube (B, B). The conceptual level demonstrated during the study of the first transformation developed from the first category to the second (C1-C2), whilst that demonstrated in relation to the second transformation developed from the second category to the third (C2-C3). The student considered first mental transformation of the initial solid into an orthogonal parallelepiped in a rather intuitive way. However, in her attempt to transform the solid into a cube, she started to consider the alterations resulting from the specific modified properties. One such alteration was the change of the form of the faces to square. The student again used a physical referent, the dimensions of which were different, to justify her opinion regarding the transformation that caused its faces to become square. She proposed that the equivalent enlargement of the edges was a prerequisite for the change to the faces.

$R$ : Well, I want you to make the orthogonal parallelepiped you want. Can you imagine the changes that you have to make?

St13: I will make it higher.

$R$ : How are you going to make it higher?

St13: Like this? [simulating the raising of the above face using her palm].

$R$ : What are you going to change?

St13: The edge.

$R$ : Which edge? 
St13: This and this one [pointing two of edges of the height dimension].

$R:$ If you change these edges how it will be?

St13: No, we have to change the other two as well ... We will raise this one, this one, this on and this one...

$R$ : How much will you raise them?

St13: Let's say 3.

$R$ : Why do you want to make this change?

St13: To become square.

$R:$ What?

St13: These faces [pointing the four faces].

\subsubsection{Building Relations among the Solids Based on the Modified Properties}

A number of children reached the most advanced level of thinking about a geometrical solid. Initially considering the transformation of the orthogonal parallelepiped through the modification of its angles, student St3 focused on the specific modified angles, though he related these changes to the formation of the whole solid (C2). At the next stage of the experiment, he related the specific modified angles to the indirect change in the volume of the solid.

St3: Two of its faces and its angles will be modified.

$R$ : Will anything else?

St3: It will simply become oblique.

$R$ : What else will change?

St3: Aha! Its volume will become smaller.

$R$ : Why do you believe that its volume becomes smaller? What do you change?

St3: Its angles.

R: How much do we have to change the angles?

St3: As much as we want. It could go there [using his palms, he represents an extreme transformation where the solid becomes flat].

$R$ : If it reaches there, will the solid exist?

St3: It becomes flat.

$R:$ So, what will happen to the volume?

St3: It will have no volume. It becomes a plane.

The justification for considering the reduction in volume as a result of the modification of the angles was based on the student's experience in the classroom teaching experiment with the dynamic model of orthogonal parallelepiped, which allowed a gradual transformation of the solid into a series of different solids.

The initial orthogonal parallelepiped became oblique and finally flat. The students developed an intuitive appreciation of volume through the comparison of this series of solids using a dynamic physical model of an orthogonal parallelepiped during the classroom teaching experiment (Markopoulos \& Potari, 2000; Markopoulos, 2003). St3 seemed to transfer this kind of experience, and adapt this approach to the case of mental transformations.

\section{Conclusion}

The role of the dynamic transformation of solids is crucial in the development of children's thinking concerning the geometrical solid. As the children focused on the process of the transformation, they seemed to become aware of the role of the "direct" and the "indirect" or subsequent modified properties on the formation of the solid. The development of their thinking from a holistic consideration to an understanding of the abstract relationship between the properties and the solid involves two intermediate levels of thinking: 1) an initial intuitive understanding of the relationship between the direct modified properties and the solid; and 2) a subsequent development of awareness of the indirect changes the direct ones cause to the solid during its transformation (Ambrose \& Kenehan, 2009). The role of the dynamic transformation in the development of these two intermediate levels was significant. When focusing on the transformation process, the children started to become conscious of the relationship between the properties of the solid and the effect of their modifications.

Furthermore, in the complex context of the mental manipulation of the solid, most of the children referred to a physical referent. The way it was used differed from child to child depending on their level of thinking. The 
children who conceived the solid in a rather holistic way, for example, used the physical referent as a prototypical resource in order to construct a mental image of the solid. The dynamic manipulation of this mental image was impossible, as they could not focus on its properties. The children who could only relate the solid to its specific modified properties used this physical model in the creation of the mental image as well as in the process of transformation. The children who, however, reached the third level of conceptual ability used the physical referent as an instrument in their attempt to communicate their thinking. Those children whose thinking reached the most advanced level used the physical model to exemplify the transformation process and the relationship between the subsequently modified properties and the solid. It would appear that the use of the concrete model does not necessarily imply primitive thinking, but can act as an intuitive tool that, in the process of transformation, can possibly support abstractions and formalisations to geometry education (Pittalis \& Christou, 2010; Lowrie, 2012).

The study indicated that, in the context of mental transformations, children's thinking of the three-dimensional geometrical concept could develop from a holistic to a relational consideration, but not necessarily in a linear order. Although not all the children reached an advanced level of thinking, the context of dynamic transformations promoted the development of most children's geometrical thinking. There is also an indication that children's experience with dynamic transformations of physical models in a mathematics classroom environment can probably act as a means that can allow children to transfer experience from one context to the other (Evans, 1999). Moreover, the whole process of transformation can be considered as a metacognitive activity (Pandiscio \& Orton, 1998) that can help children to become aware of their actions, either physical or mental, and lead to a deeper understanding of the concept of geometrical solid.

\section{References}

Ambrose, R., \& Kenehan, G. (2009). Children's Evolving Understanding of Polyhedra in the Classroom. Mathematical Thinking and Learning, 11, 158-176. http://dx.doi.org/10.1080/10986060903016484

Arnold, L., \& Lawson, M. (2003). Spatial Problem-Solving in Year 7 Mathematics: An Examination of the Effects of Use of a Computer-Mediated Software Program. Mathematics Education Research Journal, 15, 187-202. http://dx.doi.org/10.1007/BF03217378

Battista, M. T. (2007). The Development of Geometric and Spatial Thinking. In F. K. Lester (Ed.), Second Handbook of Research on Mathematics Teaching and Learning (pp. 843-908). Charlotte, NC: Information Age.

Battista, M. T., \& Clements, D. H. (1996). Students’ Understanding of Three-Dimensional Rectangular Arrays of Cubes. Journal for Research in Mathematics Education, 27, 258-292. http://dx.doi.org/10.2307/749365

Bell, A. (1993). Some Experiments in Diagnostic Teaching. Educational Studies in Mathematics, 24, 115-137. http://dx.doi.org/10.1007/BF01273297

Bishop, A. J. (1985). Visualizing Rectangular Solids Made of Small Cubes: Analyzing and Effecting Students’ Performance. Educational Studies in Mathematics, 16, 389-409. http://dx.doi.org/10.1007/BF00417194

Bliss, J., Monk, M., \& Ogborn, J. (1983). Qualitative Data Analysis for Educational Research. London: Croom Helm.

Brown, D. L., \& Wheatley, G. H. (1997). Components of Imagery and Mathematical Understanding. Focus on Learning Problems in Mathematics, 19, 45-70.

Cobb, P., Yackel, E., \& Wood, T. (1992). A Constructivist Alternative to the Representational View of Mind in Mathematics Education. Journal for Research in Mathematics Education, 23, 2-33. http://dx.doi.org/10.2307/749161

Cohen, N. (2003). Curved Solid Nets. In N. Paterman, B. J. Doughery, \& J. Zillox (Eds.), Proceedings of the 27th International Conference of Psychology in Mathematics Education, Vol. 2, (pp. 229-236). Hawaii: University of Hawaii.

Cooper, M., \& Sweller, J. (1989). Secondary School Students' Representations of Solids. Journal for Research in Mathematics Education, 20, 202-212. http://dx.doi.org/10.2307/749283

Duval, R. (2006). A Cognitive Analysis of Problems of Comprehension in a Learning of Mathematics. Educational Studies in Mathematics, 61, 103-131. http://dx.doi.org/10.1007/s10649-006-0400-z

Duval, R. (2011). Why Figures Cannot Help Students to See and Understand in Geometry? Analysis of the Role and the Cognitive Functioning of Visualization. In Symposium Mathematics Education Research at the University of Cyprus and Tel Aviv University (pp. 22-23). Cyprus: University of Cyprus.

Evans, J. (1999). Building Bridges: Reflections on the Problem of Transfer of Learning in Mathematics. Educational Studies in Mathematics, 39, 23-44. http://dx.doi.org/10.1023/A:1003755611058

Fischbein, E. (1993). The Theory of Figural Concepts. Educational Studies in Mathematics, 24, 139-162. 
http://dx.doi.org/10.1007/BF01273689

Freudenthal, H. (1991). Revisiting Mathematics Education. Dordrecht: Kluwer Academic.

Gutierrez, A. (1996). Visualization in 3-Dimensional Geometry: In Search of a Framework. In L. Puig, \& A. Gutierrez (Eds.), Proceedings of the 20th International Conference for the Psychology of Mathematics Education (Vol. 1, pp. 3-19). Valencia: Universidad de Valencia.

Hershkowitz, R. (1990). Psychological Aspects of Learning Geometry. In P. Nesher, \& J. Kilpatrick (Eds.), Mathematics and Cognition: A Research Synthesis by the International Group for the Psychology of Mathematics Education (pp. 70-95). Cambridge: Cambridge University Press.

Hershkowitz, R. (1989). Visualization in Geometry-Two Sides of the Coin. Focus on Learning Problems in Mathematics, 11, 61-76.

Hunting, R. P. (1997). Clinical Interview Methods in Mathematics Education Research and Practice. Journal for Research in Mathematics Education, 16, 145-165. http://dx.doi.org/10.1016/s0732-3123(97)90023-7

Jones, K. (2000). Providing a Foundation for Deductive Reasoning: Students’ Interpretations When Using Dynamic Geometry Software and Their Evolving Mathematical Explanations. Educational Studies in Mathematics, 44, 55-85. http://dx.doi.org/10.1023/A:1012789201736

Lakoff, G., \& Núñez, R. E. (2000). Where Mathematics Comes from. How the Embodied Mind Brings Mathematics into Being. New York: Basic Books.

Lawrie C., Pegg, J., \& Gutierrez, A. (2000). Coding the Nature of Thinking Displayed in Responses on Nets of Solids. In T. Nakahara, \& M. Koyama (Eds.), Proceedings of the 24th International Conference for the Psychology of Mathematics Education (Vol. 3, pp. 215-222). Hiroshima: ERIC.

Lawrie, C., Pegg, J., \& Gutierrez, A. (2002). Unpacking Student Meaning of Cross-Sections: A Frame for Curriculum Development. In A. D. Cockburn, \& E. Nardi (Eds.), Proceedings of the 26th International Conference for the Psychology of Mathematics Education (Vol. 3, pp. 289-296). Norwich: SIME.

Lean, G., \& Clements, M. A. (1981). Spatial Ability, Visual Imagery, and Mathematical Performance. Educational Studies in Mathematics, 12, 267-299. http://dx.doi.org/10.1007/BF00311060

Lowrie, T. (2012). Visual and Spatial Reasoning: The Changing Form of Mathematics Representation and Communication. In B. Kaur, \& T. T. Lam (Eds.), Reasoning, Communication and Connections in Mathematics, Yearbook 2012, Association of Mathematics Teachers (pp. 149-168). Singapore: Word Scientific Publishing.

Ma, H. L., Wu, D. B., Chen, J. W., \& Hsieh, K. J. (2009). Mitchelmore's Development stages of the Right Rectangular Prisms of Elementary School Students in Taiwan. In M. Tzekaki, M. Kaldrimidou, \& H. Sakonidis (Eds.), Proceedings of the 33rd Conference of the International Group for the Psychology of Mathematics Education (Vol. 4, pp. 57-64). Thessaloniki: PME.

Mariotti, M. A. (1989). Mental Images: Some Problems Related to the Development of Solids. In G. Vergnaud, J. Rogalski, \& M. Artique (Eds.), Proceedings of the 13th International Conference for the Psychology of Mathematics Education (Vol. 2, pp. 258-265). Paris: PME.

Mariotti, M. A. (2000). Introduction to Proof: The Mediation of a Dynamic Software Environment. Educational Studies in Mathematics, 44, 25-53. http://dx.doi.org/10.1023/A:1012733122556

Markopoulos, C. (2003). Children’s Thinking of Geometrical Solids in a Computer-Based Environment. In T. Triandafillidis, \& K. Hatzikiriakou (Eds.), Proceedings of the 6th International Conference on Technology in Mathematics Education (pp. 152-157). Volos: SIME.

Markopoulos, C., \& Potari, D. (2000). Dynamic Transformations of Solids in the Mathematics Education. In T. Nakahara, \& M. Koyama (Eds.), Proceedings of the 24th International Conference for the Psychology of Mathematics Education, (Vol. 3, pp. 215-222). Hiroshima: ERIC.

Markopoulos, C., \& Potari, D. (1999). Forming Relationships in Three Dimensional Geometry through Dynamic Environments. In O. Zaslavsky (Ed.), Proceedings of the 23rd International Conference for the Psychology of Mathematics Education (Vol. 3, pp. 273-280). Haifa: PME.

Mitchelmore, M. C. (1980). Prediction of Developmental Stages in the Representation of Regular Space Figures. Journal for Research in Mathematics Education, 11, 83-93. http://dx.doi.org/10.2307/748901

Modenov, P. S., \& Parkhomenko, A. S. (1965). Geometric Transformations, Euclidean and Affine Transformations (Vol. 1). New York: Academic Press.

Otte, M. (1997). Mathematics, Semiotics, and the Growth of Social Knowledge. For the Learning of Mathematics, 17, 47-54.

Pandiscio, E., \& Orton, R. E. (1998). Geometry and Metacognition: An Analysis of Piaget's and van Hiele’s Perspectives. Focus on Learning Problems in Mathematics, 20, 78-87. 
Pegg, J. (1997). Broadening the Descriptors of van Hiele’s Levels 2 and 3. In Proceedings of the 20th Annual Meeting of the Mathematics Education Research Group of Australasia (pp. 391-396). Hamilton: MERGA.

Pegg, J., \& Baker, P. (1999). An Exploration of the Interface between van Hiele’s Levels 1 and 2: Initial Findings. Environments. In O. Zaslavsky (Ed.), Proceedings of the 23rd International Conference for the Psychology of Mathematics Education (Vol. 4, pp. 25-32). Haifa: PME.

Piaget, J. (1972). Psychology and Epistemology. Towards a Theory of Knowledge. Westminster: Penguin Books.

Piaget, J., \& Garcia, R. (1989). Psychogenesis and the History of Science. New York: Columbia University Press.

Pittalis, M., \& Christou, C. (2010). Types of Reasoning in 3D Geometry Thinking and Their Relations with Spatial Ability. Educational Studies in Mathematics, 75, 191-212. http://dx.doi.org/10.1007/s10649-010-9251-8

Potari, D., \& Spiliotopoulou, V. (1992). Children's Representations of the Development of Solids. For the Learning of Mathematics, 12, 38-46.

Presmeg, N. (2006). Research on Visualization in Learning and Teaching Mathematics. In A. Gutiérrez, \& P. Boero (Eds.), Handbook of Research on the Psychology of Mathematics Education: Past, Present and Future (pp. 205-236). Rotterdam: Sense.

Sack, J. J. (2013). Development of a Top-View Numeric Coding Teaching-Learning Trajectory within an Elementary Grades 3-D Visualization Design Research Project. The Journal of Mathematical Behavior, 32, 183-196. http://dx.doi.org/10.1016/j.jmathb.2013.02.006

Sdrolias, K. A., \& Triandafillidis, T. A. (2008). The Transition to Secondary School Geometry: Can There Be a "Chain of School Mathematics”? Educational Studies in Mathematics, 67, 159-169. http://dx.doi.org/10.1007/s10649-007-9093-1

Stylianou, D. A., Leikin, R., \& Silver, E. A. (1999). Exploring Students' Solution Strategies in Solving a Spatial Visualization Problem Involving Nets. In O. Zaslavsky (Ed.), Proceedings of the 23rd International Conference for the Psychology of Mathematics Education (Vol. 4, pp. 241-248). Haifa: PME.

Van Hiele, P. M. (1986). Structure and Insight. A Theory of Mathematics Education. London: Academic Press.

Waldegg, G. (2004). Problem Solving, Collaborative Learning and History of Mathematics: Experiences in Training in-Service Teachers. Mediterranean Journal for Research in Mathematics Education, 3, 63-72.

Wheatley, G. H. (1990). Spatial Sense and Mathematics Learning. Arithmetic Teacher, 37, 10-11. 